\title{
L'« uberisation » de la logistique : disruption ou continuité ? Le cas de l'Île-de-France
}

\section{The uberization of logistics: disruption or continuity? The case of the} Greater Paris region

\author{
Pierre Vétois ${ }^{1}$, Nicolas Raimbault ${ }^{2}$ \\ ${ }^{1}$ Responsable des partenariats FretLink, pierre.vetois@fretlink.com \\ ${ }^{2}$ Department of Urban Development and Mobility, Luxembourg Institute of Socio-Economic Research (LISER), \\ Luxembourg, nicolas.raimbault@liser.lu
}

\begin{abstract}
RÉSUMÉ. Cet article analyse de manière empirique les conséquences d'une "uberisation » de la logistique, en se concentrant sur le cas francilien. Nous nous appuyons sur une série d'entretiens semi-directifs avec un panel d'acteurs de cette transformation, fondateurs de startups, dirigeants d'entreprises, coursiers, ainsi que sur une base de données que nous avons constituée au sujet de ces acteurs. Nous montrons que la dynamique d'uberisation se concentre sur la partie la plus urbaine des chaînes logistiques, les livraisons, tandis qu'elle n'a qu'un impact limité sur la logistique des grands entrepôts situés en grande couronne. Les conséquences sociales et sociologiques dépassent la simple remise en cause du salariat. Loin de ne bouleverser que le quotidien déjà dégradé des chauffeurs-livreurs, ces derniers étant souvent sous-traitants, c'est au niveau des professions intermédiaires et des dirigeants que des changements majeurs pourraient avoir lieu. Cette dynamique approfondirait la fragmentation sociale et territoriale de la logistique francilienne entre activités uberisées et celles plus traditionnelles.

ABSTRACT. This article analyzes, in an empirical way, the consequences of an "uberization" of logistics, based on the case of the île-de-France region. Our work is based on several in-depth interviews with some of the actors of this transformation, startups founders, firm directors, couriers, and also based on a database which we have developed about said actors. This approach enables us to understand both the current transformations and the actors' logic which underpin these dynamics. We demonstrate that uberization dynamics are focusing on the most urban part of the logistic chains and the deliveries, while its impact on the part corresponding to the huge suburban distribution centers is very limited. This phenomenon would have social and sociological consequences which impact not only delivery drivers (the latter often being subcontractors) but also intermediary jobs and top managers. This dynamic would increase the territorial and social fragmentation of the logistics system between "ubered" logistics activities and more traditional logistic ones.
\end{abstract}

MOTS-CLÉS. uberisation, logistique, disruption, continuité, île-de-France, emplois, livraison.

KEYWORDS. uberization, logistics, disruption, continuity, île de France region, jobs, deliveries.

JEL: O33; R40, Z130.

\section{Introduction}

« Tout le monde commence à craindre de se faire uberiser. C'est l'idée qu'on se réveille soudainement en découvrant que son activité historique a disparu. » (Maurice Lévy, PDG du Groupe Publicis au Financial Times en décembre 2014)

Cette crainte peut être résumée par la certitude qu'aujourd'hui un secteur mûr, où les marges sont généreuses et où les acteurs principaux sont solidement établis, peut être renversé du jour au lendemain par une innovation radicale, une « disruption » (Christensen, 2013). Une innovation disruptive est une innovation (technologique, organisationnelle ou servicielle) qui, par son caractère radicalement nouveau, bouleverse un secteur au point de remettre en question les positions de force des entreprises historiquement implantées ${ }^{1}$. Ce concept est adossé à celui de « destruction créatrice » de Schumpeter

\footnotetext{
${ }^{1}$ Christensen explique que « La disruption change un marché non pas avec un meilleur produit - c'est le rôle de l'innovation pure -, mais en l'ouvrant au plus grand nombre », propos recueillis par Philippe Mabille : " La disruption est une transformation irréversible (c) 2017 ISTE OpenScience - Published by ISTE Ltd. London, UK - openscience.fr 
pour qui l'innovation ne peut venir que d'une première phase de table rase, de destruction, prélude nécessaire à la création (Schumpeter, 1942).

L'uberisation fait partie de ces disruptions. C'est un terme nouveau et encore mal stabilisé. Celle-ci consiste principalement en un émiettement du travail devenant « à la demande » et mobilisable rapidement grâce à des outils numériques. Elle contourne le salariat en répondant à des besoins ponctuels par une main d'œuvre ponctuelle. Le travailleur est ainsi mobilisé au coup par coup (Filippova, 2016). Des entreprises issues des nouvelles technologies proposent ainsi d'allouer de façon optimale l'offre à la demande par l'intermédiaire de leur plateforme numérique qui utilise des algorithmes avancés. Pour ces entreprises, il ne s'agit pas d'employer des salariés offrant ces services, mais des indépendants qui louent leurs services quand ils le souhaitent. A la clef, plus de souplesse pour eux, mais aussi moins de protection sociale, mutations que le législateur peine à prendre en compte (Soufron, 2015). D'autres inconvénients en découlent pour ces travailleurs : revenus incertains et fortement fluctuants, dépendance économique vis-à-vis de l'entreprise plateforme qui amène à un salariat déguisé, émiettement du travail qui peut aboutir à une perte de sens de celui-ci ${ }^{2}$.

Après l'hôtellerie, les taxis, viendrait le temps de la logistique à laquelle s'intéressent de plus en plus d'entreprises dites, abusivement, de l'" économie collaborative » et de nouvelles startups du numérique. La logistique, vaste domaine qui regroupe de très nombreuses activités, est-elle sur le point d'être «uberisée »?

La logistique constitue une fonction essentielle pour la bonne marche des métropoles modernes. Elle les approvisionne en matières premières et biens de consommation, leur permet d'exporter leurs productions, voire leurs déchets. Elle participe au phénomène de métropolisation (Dablanc et Frémont, 2015 ; Hesse, 2008) et se compose de nombreux sous-secteurs (entreposage, transport express, messagerie, commission de transport) qui ont chacun leurs spécificités. A cette diversité d'activités, répond aussi une diversité d'emplois, principalement ouvriers. La logistique façonne, de plus, le paysage urbain contemporain par ses entrepôts et ses importants flux routiers. Dans ce contexte, notre objectif est double.

Tout d'abord, d'un point de vue empirique, nous souhaitons saisir le poids de l'uberisation en cours des activités logistiques en Île-de-France, et cela tant d'un point de vue social qu'économique et territorial. Or, la logistique francilienne est déjà le théâtre d'une concurrence acharnée entre les entreprises de ce secteur. C'est un secteur où le travail est souvent précaire et où les conditions de travail peuvent être difficiles. La logistique francilienne est fortement marquée par la sous-traitance et même le travail non déclaré. Une fois établi un constat objectif du secteur, nous traiterons de l'impact que cette potentielle uberisation aurait sur les emplois et les organisations logistiques ainsi que ses impacts territoriaux.

Ensuite, ces premiers éléments dessinent un questionnement plus théorique. Le cas de la logistique permet d'interroger la profondeur de la « disruption» engendrée par le processus d'uberisation. Alors que les thuriféraires de l'uberisation voient en elle une rupture radicale, que peut-elle signifier dans un secteur marqué par la sous-traitance généralisée engendrant des emplois aux conditions de travail d'ores et déjà difficiles et dégradées. En ce sens, n'y aurait-t-il pas tout autant continuité que disruption dans ce contexte-là ?

\footnotetext{
du capitalisme "), La Tribune, 10 mars 2014 [URL : http://www.latribune.fr/blogs/inside-davos/20140310trib000819144/-ladisruption-est-une-transformation-irreversible-du-capitalisme-clayton-christensen.html ; consulté le 15/09/2016]

${ }^{2}$ Sur ce point, se référer aux travaux désormais classiques de G. Friedman (1956).

(c) 2017 ISTE OpenScience - Published by ISTE Ltd. London, UK - openscience.fr 
Notre démarche sera avant tout exploratoire et repose sur une actualité sans cesse changeante ${ }^{3}$. Il s'agira de mettre en évidence les principales problématiques en jeu derrière une éventuelle uberisation de la logistique francilienne et les tendances qu'il est déjà possible de distinguer. Plus précisément, nous nous appuyons sur une série d'entretiens semi-directifs avec un panel d'acteurs de cette transformation : fondateurs de startups, dirigeants d'entreprises, coursiers (tableau 2). Cette méthode permet à la fois de comprendre les mutations en cours et les logiques d'acteurs qui portent ces dynamiques. Parallèlement, nous avons constitué une base de données sur ces startups et leurs fondateurs afin d'en saisir tant les traits généraux que les spécificités (tableau 3).

Dans ce but, nous présenterons dans une première partie les grands traits de la logistique francilienne. Nous exposerons ensuite dans une seconde partie les prémisses du processus d'uberisation de la logistique en Île-de-France. Les impacts socio-économiques feront l'objet d'une troisième partie.

\section{La logistique francilienne : croissance et éclatement}

L'Ile-de-France est le principal pôle logistique français, en raison de son poids démographique et économique (Dablanc et Frémont, 2015). Avec 375000 emplois, ce qui représenterait $7 \%$ des emplois salariés franciliens (Insee, 2015), la logistique en Ile-de-France est ainsi un secteur majeur de l'emploi francilien. Ce secteur n'est toutefois pas homogène. Deux grands types d'activités logistiques se distinguent, reposant sur des géographies, des organisations économiques et des emplois bien distincts.

\section{Entrepôts périurbains et livraisons urbaines}

Une distinction assez forte apparait entre la grande logistique périurbaine et la logistique des livraisons urbaines. La première traite les marchandises au sein de grands entrepôts, dits aussi plateformes logistiques, et privilégie les transports par poids lourds faisant des liaisons interrégionales voire internationales. La seconde se charge des derniers maillons de la chaîne logistique (ou des premiers) jusqu'au client final, souvent en milieu urbain et qui peut être un particulier à son domicile (Durand et al, 2010).

Ce partage géographique du travail logistique tend à se renforcer. En effet, les activités d'entreposage quittent de plus en plus nettement les zones urbaines denses franciliennes au profit de zones plus périphériques, voire au-delà des limites de l'Île-de-France, où le foncier est moins onéreux. Ainsi, sur la période 1985-2009, les départements de grande couronne ont absorbé 45,1\% des constructions d'entrepôts, ceux limitrophes à l'Ile-de-France 37,5\%, quand Paris et la petite couronne totalisent seulement $17,4 \%$ des constructions sur cette période (Raimbault, 2014). Parallèlement, les besoins en services logistiques des centres urbains ne cessent de croître, notamment avec l'émergence de nouveaux modes de consommation qui intensifient les flux. Il s'agit notamment du e-commerce. Celui-ci explose depuis le début des années 2000. Il représentait 700 millions de transactions à l'échelle nationale en $2015^{4}$. La logistique doit répondre aux défis que cela lui pose, via à la fois la construction de nouveaux entrepôts dans les couronnes périurbaines (le e-commerce est devenu le principal moteur de la croissance de l'immobilier logistique ${ }^{5}$ ) et dans le domaine des livraisons à domicile, majoritairement en zone dense (46\% des colis étaient livrés à domicile en 2014 à l'échelle nationale).

\footnotetext{
${ }^{3}$ En 2016, par exemple, la startup Stuart, de livraison à la demande pour les entreprises, a été mise en avant par une intense campagne médiatique, quand au cours de l'été, on a appris la liquidation judiciaire de «Take eat easy ». De nouveaux acteurs émergent sans cesse, quand d'autres disparaissent.

${ }^{4}$ Source : FEVAD, Chiffres clés 2015.

${ }^{5}$ Source : Prologis, Inside The Global Supply Chain: E-Commerce And A New Demand Model For Logistics Real Estate, juillet 2014. 
De plus, l'organisation économique de ces deux maillons de la chaîne logistique se différencie également nettement. La grande logistique articulée autour des plateformes périphériques est mise en œuvre soit par des prestataires logistiques (transport et entreposage) soit directement par des entreprises de l'industrie, de la grande distribution et du e-commerce (dites chargeurs par opposition aux prestataires). Elles donc le fait de moyennes ou grandes entreprises, souvent de dimension nationale, voire internationale.

La logistique de la livraison est majoritairement prise en charge par des entreprises de transport pour le compte des chargeurs indiqués précédemment. Cependant, ces entreprises de transport recourent de manière massive à la sous-traitance pour l'accomplissement des livraisons elles-mêmes (Rème-Harnay et al, 2014), en particulier en Ile-de-France (Jouffe, 2008). De plus, ces entreprises n'ont souvent pas de flotte de véhicules en propre. Ceux qu'ils utilisent sont seulement loués. Des acteurs majeurs comme Chronopost peuvent sous-traiter jusqu'à $90 \%$ de leur activité. Celle-ci est confiée à de petites entreprises de transport routier, TPE voire artisans, utilisant massivement des véhicules utilitaires légers (VUL). 94\% des entreprises de transport léger (en VUL) ont moins de 5 salariés et $55 \%$ n'en ont pas du tout. Ces entreprises ont une trésorerie faible (n'ayant pas de capacités d'investissement, ils utilisent des VUL souvent anciens et polluants) et des moyens de négociations qui le sont encore plus. Dans près de $75 \%$ des cas, ces sous-traitants ne travaillent qu'avec un seul donneur d'ordre, ce qui réduit encore leurs marges de manœuvre en termes de négociation (Rème-Harnay, 2012). Grâce à leur position très dominante, des géants comme Chronopost ou UPS signent avec ces sous-traitants des contrats aux conditions léonines qui mettent ces derniers dans des situations de dépendance quasi totale. Enfin, pour les donneurs d'ordre, la sous-traitance leur permet non seulement d'éviter des embauches, mais, de plus, le très grand nombre de sous-traitants qui travaillent pour les grands donneurs d'ordre permet d'éviter les revendications collectives qui pourraient avoir lieu si tous les chauffeurs étaient leurs salariés.

\section{Les emplois logistiques entre deux mondes ouvriers}

Le taux d'ouvriers est particulièrement élevé dans le secteur de la logistique par comparaison avec les autres services marchands : $80 \%{ }^{6}$ contre $15 \%$ en moyenne. Il n'est donc pas étonnant que le salaire horaire brut médian dans la logistique soit inférieur à celui des autres services marchands ( $15 €$ contre $18 €)$ (Insee, 2015). Toutefois, ces emplois ouvriers sont relativement divers : deux grandes familles de métiers apparaissent, étroitement liées à la distinction entre livraisons urbaines et entrepôts périurbains.

D'une part, les ouvriers du tri, de l'emballage et de l'expédition, les préparateurs de commande ainsi que les caristes correspondent aux métiers des entrepôts, dont nous avons indiqué la géographie de plus en plus périphérique. Plus précisément, ces emplois ouvriers, au nombre de 700000 en France (Mariotte, 2007 ; Tranchant, 2015), sont particulièrement concentrés dans quelques pôles de la grande couronne nord, est et sud de l'Île-de-France, en plus des pôles historiques de Seine-Saint-Denis et du Val de Marne (Insee, 2015 ; figure 1).

Ces ouvriers sont majoritairement salariés des entreprises exploitant les entrepôts, tandis que le taux de recours à l'emploi intérimaire s'élèverait à $20 \%$ (Insee, 2015). Les conditions de travail de ces ouvriers se rapprochent de celles des ouvriers spécialisés de l'industrie manufacturière (Gaborieau, 2012).

\footnotetext{
${ }^{6}$ INSEE, Recensement de la population 2013. La proportion est similaire en Allemagne : Dénombrement des salariés par branche, Bundesagentur für Arbeit, 2013. 


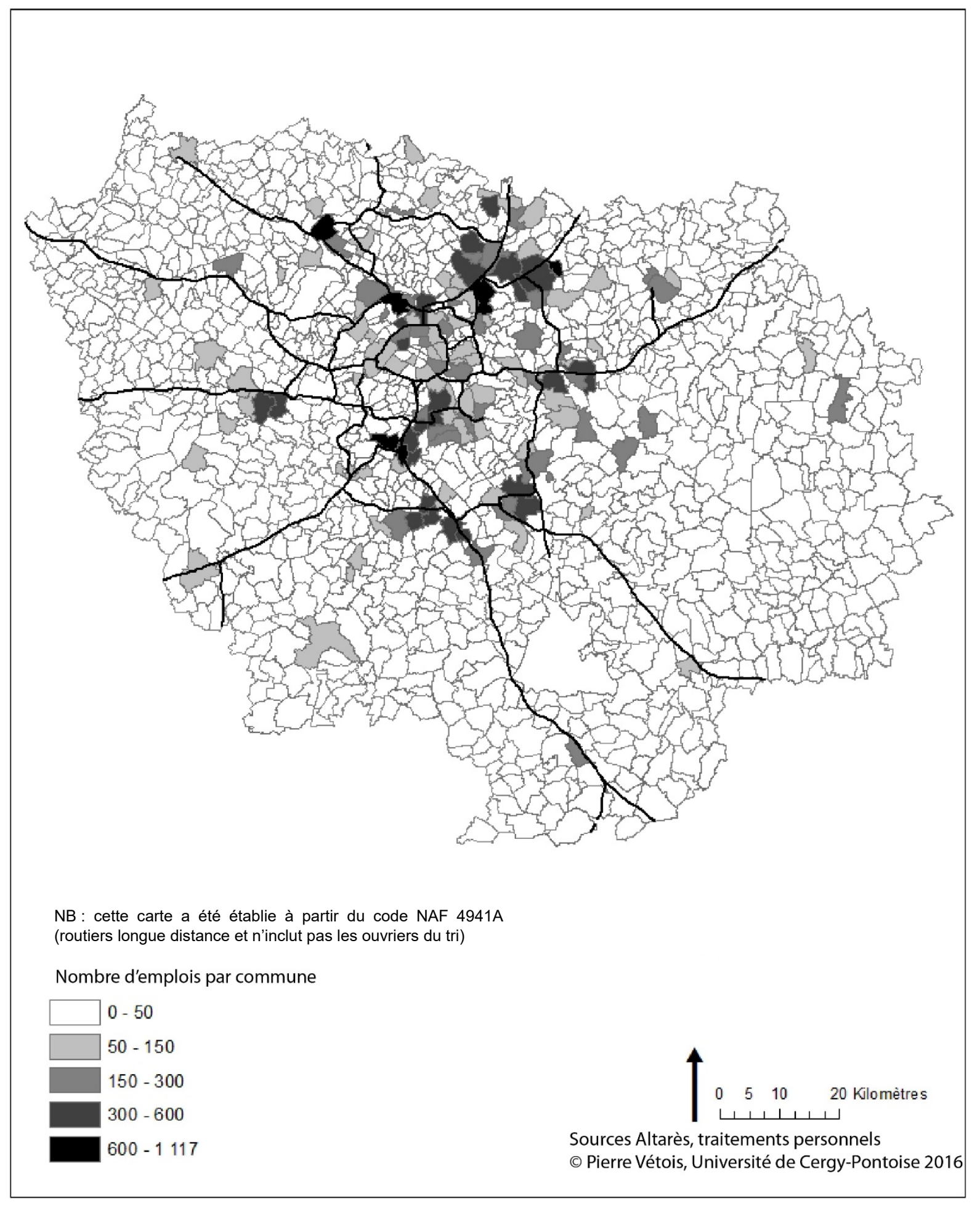

Figure 1. L'emploi de la grande logistique en Ile-de-France

De l'autre, les conducteurs routiers et grands routiers et surtout les conducteurs livreurs et les coursiers (les plus nombreux) se chargent du transport lui-même et en particulier des livraisons urbaines. A l'échelle nationale, ces métiers rassemblent entre 500000 et 600000 emplois (Mariotte, 2007 ; Tranchant, 2015).

Les chauffeurs-livreurs correspondent à environ la moitié de ces emplois. L'estimation de leur effectif est toutefois difficile à réaliser en raison des frontières mouvantes entre le transport dit régional et les livraisons (la distance moyenne d'un transport de marchandises est de $100 \mathrm{~km}$ en France : voir CGEDD, 2012) et surtout en raison de la part importante de travailleurs indépendants. En effet, 
l'organisation économique de la livraison, que nous avons exposée ci-dessus, a pour conséquence des conditions de travail souvent très dures pour les chauffeurs-livreurs (Charrin, 2013). Ceux-ci dépassent largement le nombre autorisé d'heures de travail pour réussir à livrer tous leurs colis. Les chauffeurs « décrivent des journées de plus de 12 heures de travail, parfois de 5 heures à 20 heures, six jours sur sept » selon le journaliste Dan Israël ${ }^{7}$. Ces journées très longues sont à coupler avec la manutention de colis souvent lourds qui peuvent provoquer des arrêts maladie (Libeskind, 2015).

Ces dépassements d'horaires s'accompagnent aussi de travail non déclaré. Il est bien sûr difficile d'avoir des estimations, mais il est probable que celui-ci soit très répandu, notamment en Ile-de-France (Libeskind, 2015). Le transport fait partie des secteurs les plus touchés par ce type d'activités illégales ${ }^{8}$. Enfin, d'un point de vue géographique, ces emplois sont particulièrement localisés en banlieue proche, en particulier en Seine-Saint-Denis et, dans une moindre mesure, dans le Val de Marne (figure 2).

De plus, au-delà de leur localisation administrative (qui est celle de leur entreprise), les chauffeurslivreurs travaillent très majoritairement au sein de la zone dense et notamment à Paris (en particulier dans les arrondissements centraux où sont localisées de nombreuses entreprises).

\footnotetext{
7 Source: Dan Israël, «Chronopost: les sous-traitants se disent pris à la gorge», Médiapart, 7 mars 2013. [URL: https://www.mediapart.fr/journal/france/270213/chronopost-les-sous-traitants-pris-la-gorge?onglet=full ; consulté le 02/09/2016]

8 Source: David Bensoussan, «Travail au noir, l'alerte rouge », Challenges 07/02/2014 [URL: https://www.challenges.fr/economie/travail-au-noir-l-alerte-rouge_69628; consulté le 02/06/16]. 


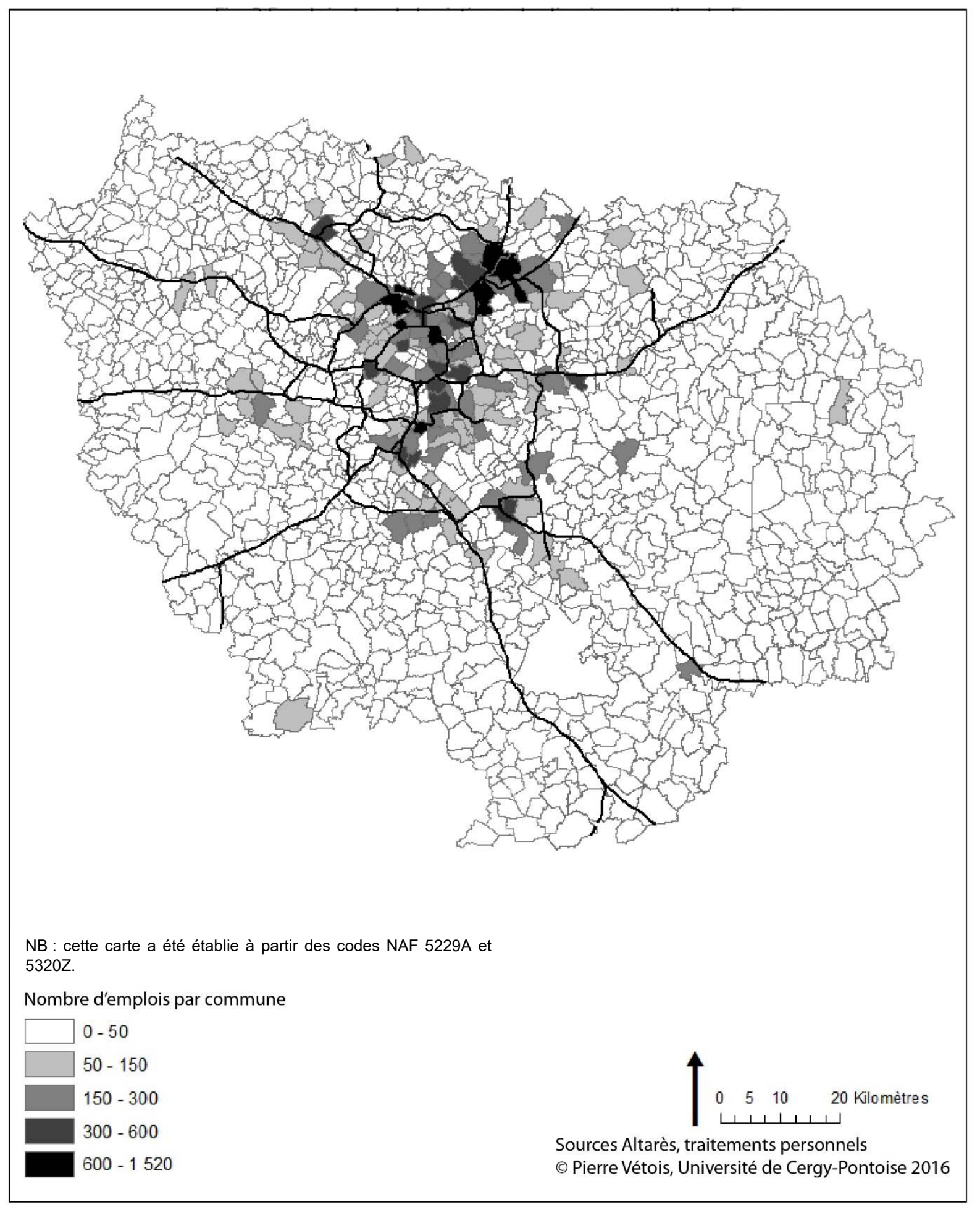

Figure 2. L'emploi de la logistique de précision en lle-de-France

Enfin, concernant à la fois la grande logistique et les livraisons urbaines, la part des cadres est historiquement faible dans la logistique. Elle serait de $9 \%$ dans l'entreposage (24\% en incluant l'encadrement intermédiaire) et de 4,5\% dans le transport routier de marchandises ( $9 \%$ en incluant l'encadrement intermédiaire) (Mariotte, 2007). En ce qui concerne les prestataires logistiques, cette part serait toutefois en constante progression entre 2004 et 2011, selon un rythme un peu plus rapide que dans les autres services marchands (Insee, 2015).

Ces distinctions géographiques et socio-économiques entre grande logistique périurbaine et logistique urbaine sont cruciales car la dynamique d'uberisation s'avère tout à fait différente selon ces deux grandes familles de métiers. 


\section{Les prémices d'une uberisation de la logistique}

En définissant l'uberisation comme une évolution qui allie usage d'applications mobiles, automatisation de tâches de gestion autrefois confiées à des humains et emploi d'indépendants nonsalariés pour réaliser des services ponctuels, il apparait de plus en plus nettement que certains services logistiques s'uberisent progressivement. Nous présenterons dans un premier temps les pionniers de cette dynamique puis nous confronterons les deux modèles économiques qui émergent: celui des startups de la livraison et celui des géants de l'internet qui se concentrent sur le dernier kilomètre. Enfin nous nous interrogerons sur le caractère disruptif de ces expériences.

\section{Les pionniers : la livraison de repas à domicile}

Les premiers services mis en place concernent la livraison à domicile de repas à vélo dans l'hyper centre des plus grandes villes françaises ${ }^{9}$.

La livraison de repas à domicile est un service ancien qui était généralement proposé par certains types de restauration eux-mêmes. Ce service a été récemment transformé et étendu à de nouveaux restaurateurs et clients par des applications comme Foodora, Take eat easy ou Deliveroo. Il s'agit de plates-formes en ligne mettant en relation des restaurateurs et des clients et recourant à des livreurs indépendants, principalement des chômeurs ou des étudiants, pour assurer les livraisons proprement dites. Elles donnent un avant-goût de ce que pourrait être une logistique du dernier kilomètre uberisée :

« Je m'inscris sur un calendrier en ligne [...] J'ai une visibilité d'un mois [...] j'utilise cette application environ deux fois par semaine »

(Entretien, étudiant, 26 ans, coursier à vélo pour Take eat easy à Lille, 26/04/2016)

Le rythme de travail est dicté par l'algorithme qui optimise les livraisons, de plus en plus nettement à l'avantage de l'entreprise : "Avant l'algorithme était assez souple, avec une ou deux livraisons par soir et on était quand même payé, maintenant c'est beaucoup plus intense ». Ainsi, dans le cas de Take eat easy, l'algorithme poussait à atteindre le taux de sept courses par livreur par soirée (18-22h) selon nos informations. D'un point de vue juridique, les livreurs sont invités à s'inscrire comme autoentrepreneurs. Pour les responsabiliser, le site les note et inflige des pénalités quand le livreur fait défaut.

\section{Des startups de la livraison pour l'urbain dense}

Ce modèle de livraison s'avère transposable à la livraison de colis même si certaines interrogations ne sont pas encore levées. La livraison par des indépendants de repas n'est ainsi pas aussi sensible que celle de colis potentiellement précieux. Un chargeur sera-t-il d'accord pour que le « transporteur » fasse livrer sa marchandise par un indépendant exerçant sur son temps libre avec tout l'amateurisme que cela pourrait supposer ? Comment ainsi se prémunir contre le vol, l'endommagement des biens avec de telles applications ? Malgré ces écueils juridiques, plusieurs tentatives de services uberisés de livraison de colis ont émergé au sein des grandes métropoles mondiales.

Deliver.ee est une plateforme numérique. Elle fonctionne comme une centrale d'achat de prestations de transport avec près de 1000 coursiers partenaires (qui peuvent être soit titulaires d'une capacité de transport $^{10}$, soit des particuliers) et 500000 euros levés en octobre 2014. Elle travaille sous le statut

\footnotetext{
${ }^{9}$ Barbedette B., « Les coursiers vélos bientôt secoués par "l'uberisation" » ? Transports Urbains, juillet 2015 [URL : http://www.wktransport-logistique.fr/actualites/detail/86418/actualites-detail-officiel-transporteurs/les-coursiers-velos-bientot-secoues-par-1uberisation.html ; consulté le 03/03/2016].

${ }^{10}$ Il s'agit d'un document obligatoire pour toute personne qui souhaite être transporteur routier de marchandises. Il faut réussir un examen pour l'obtenir. 
juridique de commissionnaire de transport avec des entreprises comme Fnac ou Nike. Deliver.ee se place dans la position d'intermédiaire entre des transporteurs très divers (professionnels ou non, à vélo, en VUL, à pied, etc...) et des consommateurs demandeurs de livraisons toujours plus rapides. L'avantage comparatif de cette entreprise réside avant tout dans ses algorithmes qui permettent d'optimiser le système de livraison en allouant au bon moment, au bon endroit, la prestation de transport la plus adaptée au besoin du consommateur. Elle gère 100 à 300 livraisons par jour dans Paris et réalise $80 \%$ de son chiffre d'affaires en Île-de-France. Une des solutions testées actuellement par Deliver.ee pour parer aux difficultés juridiques évoquées plus haut est de proposer au client de signer une procuration sur son site internet pour que le coursier aille chercher le colis en point relais. Par la suite, le coursier s'occupe de la livraison du colis, depuis le point relais jusqu'au destinataire final. La responsabilité est transférée à l'aide d'un code secret transmis par le site Deliver.ee et d'une double signature sur le «personal digital assistant » (PDA) du point relais et sur le smartphone du coursier Deliver.ee ${ }^{11}$.

Pour un de ses fondateurs (ayant aujourd'hui quitté l'entreprise), Deliver.ee est " avant tout une société de technologie et nous cherchons à faire correspondre un besoin à une compétence ${ }^{12}$. Ces startups ne disposent pas de moyens de transport en propre et ne cherchent pas plus à investir dans des sites d'entreposage, à la différence des entreprises dominant actuellement le secteur logistique telles que DHL ou Fedex.

Colisweb est une startup lilloise qui propose aux commerçants (physiques ou en ligne) de livrer leurs commandes selon des délais courts ( 2 à $3 \mathrm{~h}$ en centre-ville). Elle compte vingt-cinq clients en 2015 qui sont essentiellement des grands comptes. Elle a déjà noué des partenariats avec Café Méo ou Etam Lingerie. $75 \%$ de son chiffre d'affaires se fait en $\mathrm{B} 2 \mathrm{C}^{13}$ et $60 \%$ du chiffre d'affaires est concentré sur la Région Ile-de-France (100 livraisons par jour sur Paris et la petite couronne). Plus qu'une révolution, son fondateur parle d'une numérisation de la logistique «j'ai travaillé sur de nouvelles solutions pour les transporteurs car c'est un monde qui a peu évolué technologiquement et qui décline [...] en raison de la dématérialisation du système» ${ }^{14}$.

\section{L'intérêt croissant des géants de l'internet}

Plusieurs grands noms de l'Internet ont aussi témoigné de leur intérêt pour ce phénomène. Ainsi Amazon, Uber, Postmates, ou Google préparent des projets similaires. Amazon teste aux Etats-Unis différentes moutures d'une même idée. Que cela s'appelle «Amazon On my way», ou «Amazon Flex », l'idée est d'uberiser son dernier kilomètre. L'enjeu pour le $\mathrm{n}^{\circ} 1 \mathrm{du}$ e-commerce mondial est de réduire ses coûts de livraison qui enflent plus vite que ses profits ${ }^{15}(+31 \%$ de coûts de livraison en 2015). L'entreprise propose à des particuliers de venir retirer des colis dans des magasins partenaires, utilisés comme des points relais, et de les livrer aux destinataires finaux. Une application maison permet au livreur indépendant de se rendre au point relais pour y récupérer le colis. L'objectif est clair : s'affranchir des grands expressistes américains que sont UPS ou FEDEX qui facturent environ $8 \$$ la livraison. Amazon est, il est vrai, un cas particulier, car l'entreprise de Jeff Besos cherche une intégration la plus verticale possible de ses activités. Mais de ce fait, cette entreprise participe aussi de la disruption de la logistique en y important de nombreuses innovations.

\footnotetext{
${ }^{11}$ « Relais Colis et la start-up deliver.ee s'associent pour tester un nouveau service de livraison innovant en avant-première pour les clients La Redoute » Communiqué de presse, 10 novembre 2015

${ }^{12}$ Louniangou Ntsika C., «Deliver.ee, le Uber français de la livraison de marchandises arrive en ville », Logistique Magazine février 2015, [URL : http://www.actu-transport-logistique.fr/supply-chain/deliveree-le-uber-francais-de-la-livraison-de-marchandises-arriveen-ville-180283.php ; consulté le 25/03/2016].

${ }_{14}^{13}$ Business to Consumer : service proposé par une entreprise à destination de particuliers.

14 Valérie Abrial, « Colisweb, le pionnier de la livraison instantanée », La Tribune, 06/01/2015 [URL: http://www.latribune.fr/loisirs/la-tribune-now/20150106trib8089549e1/colisweb-pionnier-de-la-livraison-instantanee.html ; consulté le $07 / 04 / 2016]$.

${ }^{15}$ Bensinger G., “Amazon's Next Delivery Drone: You”, Wall Street Journal, juin 2015, [consulté le 10/03/2016]. 
On retrouve des expérimentations comparables avec Google Express et Uber Rush qui, contrairement à Amazon, s'affirmeraient en tant que nouveaux prestataires possibles pour les chargeurs, grâce à leur maitrise des technologies numériques. Uber propose ainsi déjà à ses chauffeurs VTC de transporter des colis aux Etats-Unis.

\section{Disruption ou mise à jour numérique de la logistique de la livraison urbaine ?}

$\mathrm{Au}$ terme de ce panorama des premiers signaux d'uberisation de la logistique, il apparait nettement que la majorité de ces initiatives se concentrent sur les derniers maillons de la chaîne logistique : la livraison, en particulier au centre des grandes villes. La livraison en ville est en effet difficile et toujours très coûteuse :le « dernier kilomètre » représente $1 \%$ de la distance, mais $25 \%$ des coûts de la chaîne logistique ${ }^{16}$. Or, ce dernier maillon est crucial pour l'approvisionnement du centre de l'̂̂le-deFrance : 150 à 180000 livraisons ont lieu chaque jour à Paris et en petite couronne. La demande pour des livraisons $\mathrm{B}_{2} \mathrm{~B}^{17}$ et $\mathrm{B} 2 \mathrm{C}$ en ville, en lien avec le développement du e-commerce, ne faiblit pas (Barba, 2011) : en croissance depuis 2005, le nombre de colis e-commerce livrés par an est passé de 300 millions en 2010 à 400 millions en $2015^{18}$. Cette dynamique représente un gisement extrêmement intéressant pour une éventuelle uberisation si de nouveaux entrants, startups ou géants d'internet, arrivent à proposer une prestation de transport de qualité pour un prix réduit en passant par des particuliers ou des professionnels uberisés. C'est pourquoi, les initiatives se concentrent sur les centresvilles des grandes métropoles. "Nous nous sommes vite rendu compte que les autres grandes villes françaises ne vont pas nous apporter la croissance suffisante et qu'il fallait assez vite envisager l'Angleterre, ou le Benelux » explique le fondateur de Deliver.ee ${ }^{19}$.

Finalement, l'analyse de ces startups de la livraison, qui proposent aujourd'hui les services les plus aboutis, pousse à nuancer le caractère disruptif de ces jeunes pousses. En effet, ces startups adoptant le statut de commissionnaire de transport, renouvèlent autant que bouleverse ce métier traditionnel. Elles peuvent être décrites comme des «mises à jour numériques» des historiques commissionnaires de transport.

\section{L’impact sur les métiers de la logistique : quelles ruptures?}

L'examen des prémisses de l'uberisation logistique indique que cette dynamique se concentrerait sur la logistique urbaine dite du dernier $\mathrm{km}$. Quels métiers sont ainsi remis en cause et selon quelles modalités?

\section{Une remise en question du chauffeur-livreur : quels impacts sur les conditions de travail ?}

Le chauffeur-livreur, en charge des livraisons, est évidemment le métier qui est directement remis en question par les innovations logistiques numériques. Les applications mobiles des entreprises comme Deliver.ee, Uber Rush ou Amazon On my way ouvriraient en effet aux particuliers ou à des indépendants la possibilité de livrer eux-mêmes des colis en Ile-de-France. Ces derniers viendraient retirer des colis en point relais ou en entrepôt de proche banlieue pour livrer des particuliers ou des professionnels en lieu et place des chauffeurs-livreurs.

Il y aurait entre 200000 et 300000 chauffeurs-livreurs salariés, en France, auxquels il convient d'ajouter les indépendants, historiquement nombreux comme nous l'avons indiqué précédemment. En

\footnotetext{
${ }^{16}$ Source : Ropital C., Glossaire du e-commerce et de la logistique, IAU IdF, [consulté le 04/04/2016].

${ }^{17}$ Business to Business: service proposé par une entreprise à destination d'autres entreprises.

18 Source : Morganti, 2013.

19 Harmant O, «Deliver.ee veut livrer le jour même dans les capitales européennes », www.frenchweb.fr , juin 2015 [URL : http://www.frenchweb.fr/deliver-ee-veut-livrer-le-jour-meme-dans-les-capitales-europeennes/196362; consulté le 01/12/2015]. 
appliquant le même ratio pour l'Île-de-France, il y aurait près de 70000 chauffeurs-livreurs. Au-delà de ces chiffres, quelle est la portée de cette remise en cause ?

Nous avons précédemment mis en évidence combien cette profession est aujourd'hui d'ores et déjà marquée par des conditions de travail difficiles et par un très fort taux de sous-traitance. La prégnance de la sous-traitance dans le secteur du transport léger découle de deux principales caractéristiques de cette activité. Tout d'abord, son activité n'est pas très technique : la plupart des tournées sont réalisées, en centre-ville, en VUL pouvant être conduit avec un simple permis B. Ensuite, elle n'implique pas un fort investissement financier : les véhicules peuvent être loués et il n'est pas nécessaire de posséder un entrepôt, ce dernier étant exploité par les donneurs d'ordre. C'est pourquoi, cette profession attire aujourd'hui de nombreux jeunes peu qualifiés, qui ne mesurent pas toujours les coûts qu'implique cette activité. Finalement, ce sont ces mêmes caractéristiques qui font des transports légers un secteur « mûr » pour l'uberisation. En d'autres termes, le secteur du dernier kilomètre est ainsi très proche d'une situation d'uberisation de fait sans la dimension technologique. C'est cette dimension technologique que les startups et les géants d'internet sont en train d'apporter.

C'est pourquoi, il est permis de s'interroger sur l'impact social de l'uberisation de la logistique de la livraison dans la mesure où la situation actuelle est déjà profondément marquée par le contournement du salariat à travers la sous-traitance. Ainsi où est la différence profonde entre l'artisan chauffeurlivreur d'aujourd'hui travaillant avec un seul donneur d'ordre et qui est payé au nombre de colis livré et un indépendant qui ne travaillerait qu'avec une seule application et qui serait payé à la tâche ? Les deux ne sont pas salariés du donneur d'ordre et doivent s'assurer de l'entretien de leur véhicule qui est intégralement à leur charge. Les deux peuvent recevoir des pénalités en cas de retard et sont dans un rapport de stricte dépendance par rapport à l'entreprise qui leur donne du travail. De même, les deux ne vont pas compter leurs heures pour atteindre un semblant de rentabilité. L'uberisation n'est toutefois pas anodine pour le quotidien des livreurs. L'arrivée de ces applications permet un meilleur contrôle encore des livreurs dont les performances sont suivies en temps réel et leurs sont communiquées sous forme de bilan mensuel :

«Chaque mois, Take eat easy m'envoie mes statistiques sur mon profil en ligne, [...] j'y retrouve mon nombre de courses, ma vitesse moyenne, etc... C'est très complet ! »

(Entretien étudiant, 26 ans, coursier à vélo pour Take eat easy à Lille, 26/04/2016).

De plus, selon nos observations personnelles (nécessairement lacunaires), nous estimons que les nouveaux coursiers ont un profil assez différent des «anciens» du métier. Il s'agit davantage d'étudiants ou de chômeurs ne travaillant de cette façon qu'à temps partiel. En effet, il est difficile de travailler à temps plein dans le cadre de ces plateformes. L'uberisation de la logistique s'inscrit donc dans une logique de cumul d'emplois pour ces catégories de la population.

L'uberisation apparaît, ainsi, avant tout comme l'accomplissement final d'une tendance lourde d'externalisation qui a commencé par le développement de la sous-traitance auprès d'indépendants et qui se poursuivrait aujourd'hui par la mobilisation d'indépendants via des applications mobiles (Teboul, 2016). Ce résultat sur la continuité autant que sur la disruption sous-jacente à la dynamique d'uberisation rejoint l'idée de "mise à jour numérique» de la logistique à laquelle l'analyse des startups concernées nous a conduits. 
- Indépendants franchisés avec le statut d'artisans livreurs ou d'autoentrepreneur

- Activité peu technologique.

- Faible rentabilité pour les livreurs

- Travail non-déclaré courant

- Personnes issues des quartiers populaires
- Indépendants franchisés avec le statut d'autoentrepreneur partenaires d'une plateforme d'intermédiation

- Faible rentabilité pour les livreurs

- Suivi en temps réel des performances

- Moindre intérêt du travail nondéclaré

- Personnes issues des quartiers populaires

- Indépendants franchisés avec le statut d'artisans livreurs ou d'autoentrepreneur

- Activité peu technologique

Coursier à vélo
- Activité principalement pratiquée par des "passionnés du vélo"
- Indépendants franchisés avec le statut d'autoentrepreneur partenaires d'une plateforme d'intermédiation

- Faible rentabilité pour les livreurs

- Suivi en temps réel des performances

- Activité exercée par les plus précaires dans une logique de cumul d'emplois (étudiants, chômeurs et immigrés)

Tableau 1. Impact de l'uberisation sur le travail des chauffeurs-livreurs et des coursiers à vélo

Les entreprises de chauffeurs-livreurs traditionnelles ont ainsi un modèle d'ores et déjà fortement externalisés avec de nombreux franchisés. Les nouvelles entreprises ont un modèle qui se veut plus contemporain, mais qui est en réalité très similaire avec un système de commission à la course. Pour les coursiers à vélo, l'idée est la même avec des indépendants payés à la course et dont les entreprises récupèrent une commission.

\section{Une nouvelle querelle des Anciens et des Modernes?}

Les acteurs du secteur sont conscients qu'une uberisation est possible à court ou moyen terme et tentent de réagir en mettent en place une résistance. Apparaissent des réactions qui pourraient être les prémices d'une contestation ressemblant à celles des syndicats de chauffeurs de taxi en 2015. Un syndicat est particulièrement en pointe : le SNTL (transporteurs légers souvent axés sur le dernier kilomètre) fait ainsi régulièrement part de ses inquiétudes face à ce qu'il appelle les «pratiques déviantes de l'économie collaborative ». Le président de ce syndicat est le directeur général de Stars Service, l'un des plus grands transporteurs légers français ${ }^{20}$. Son communiqué de presse de mars 2016 est à ce titre très éclairant :

\footnotetext{
${ }^{20}$ Barbedette B., « Transports légers : Hervé Street nouveau président du SNTL »www.WKTransport.com, février 2016 [URL : http://www.wk-transport-logistique.fr/actualites/detail/91093/actualites-detail-officiel-transporteurs/transports-legers-herve-streetnouveau-president-du-sntl.html ; consulté le 03/03/2016]. 
«Après les TAXIS et les VTC, le SNTL se mobilise en force et déclenche des procédures judiciaires pour dénoncer l'anarchie qui se développe dans les métiers du Transport en général et des Marchandises en particulier. Par ces actions, le SNTL souhaite clairement rappeler aux nouveaux entrants de l'économie collaborative :

\section{Que tout n'est pas permis!}

2. Qu'ils doivent respecter les lois et règlementations en vigueur de commissionnaires ou transporteurs ;

3. Que pour livrer des colis, il est interdit de faire travailler des particuliers, des autoentrepreneurs autres que des vélos, des sociétés de transport de personnes...

4. Que les fonds d'investissement ou acteurs financiers qui les financent sans réserve s'exposent aux mêmes risques de sanctions qu'eux. » (Communiqué de presse du SNTL, 16 mars 2016)

En réalité, le SNTL ne défend pas réellement les conditions de travail des chauffeurs, mais plutôt la position dominante de ses membres sur le marché du transport léger et. En effet, les livreurs sont aujourd'hui pris en tenaille par les injonctions contradictoires d'une métropole parisienne qui se rêve sans voitures, de consommateurs demandeurs de livraisons à domicile quasiment gratuites et instantanées et de donneurs d'ordre optimisant au maximum les courses et les tournées.

Le cœur du problème est de définir l'identité juridique des startups logistiques. Comme cela a été expliqué plus haut, celles-ci se définissent comme des entreprises de nouvelles technologies, comme de simples intermédiaires entre les clients et les transporteurs. Les professionnels du secteur les accusent d'être en réalité des commissionnaires de transport déguisés (qui sont, eux, légalement des intermédiaires de transport). Ce statut est juridiquement défini et par là même encadré. Son principal «défaut» aux yeux des jeunes entrepreneurs est l'obligation de résultat, inscrite dans la loi, pour le commissionnaire qui est totalement responsable de la marchandise qu'il fait transporter. Or, c'est très exactement l'inverse que choisissent les startups : pour elles, elles ne sont pas responsables et ne font que «mettre en relation». Certaines ont remis au goût du jour un ancien statut juridique, celui d'auxiliaire de transport qui est très proche de celui de commissionnaire, mais comporte néanmoins moins d'obligations.

Ces nuances juridiques posent une question de fond. Les startups qui ont décidé de jouer selon les règles traditionnelles en adoptant le statut de commissionnaire de transport ne sont pas récompensées de leur effort: elles ont plus de mal à lever des fonds auprès des investisseurs (réticents devant obligation de résultats). Le système de venture capital et des business angels entre aussi en collision frontale avec le système réglementaire de la livraison. Ces investisseurs peuvent donc amener à une certaine déstabilisation déjà étudiée (Levratto et Tessier, 2016) qui n'est pas le seul fait des startupers.

\section{Sociologie d'une disruption : qui sont les « barbares » qui attaquent la logistique ?}

D'un point de vue sociologique, les acteurs de cette disruption sont des membres de l'élite intellectuelle et économique. Issus des meilleurs établissements scolaires français et/ou internationaux, ces hommes blancs (en très nette majorité) perpétuent une position dominante qui n'est pas remise en cause par la disruption économique (figure 3). 


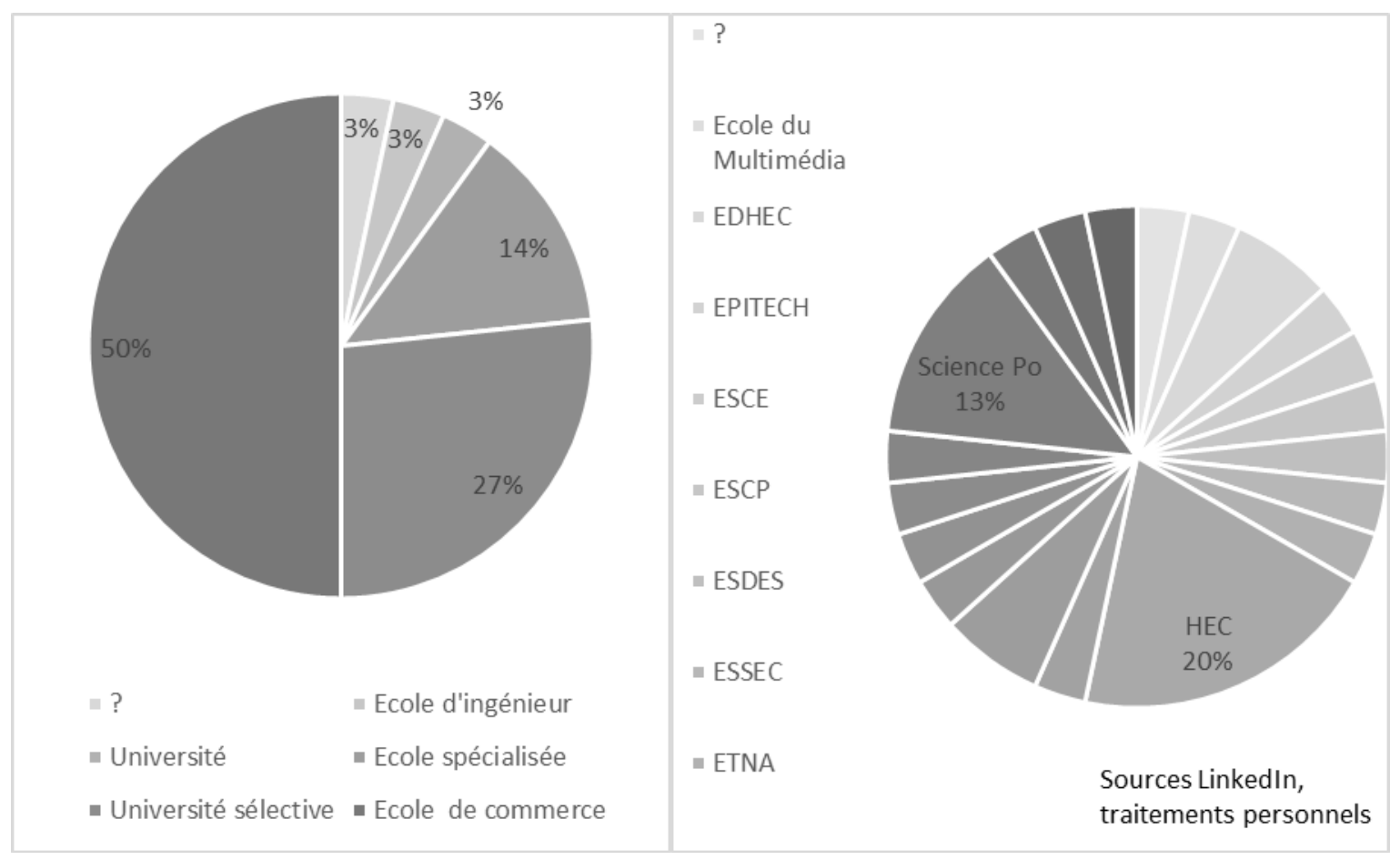

Figure 3. Formation des startupers

L'analyse nous renseigne sur la jeunesse relative de ces startupers qui s'inscrit dans la dynamique plus générale de rajeunissement de l'entreprenariat français (Boutillier, Tiran, 2016). Un tiers d'entre eux étaient en stage au titre de leur avant-dernière activité, stage qui était compris dans leur scolarité. Cette proportion tombe néanmoins à $13 \%$ pour la dernière activité. Le startuper est donc relativement jeune (même si nous ne pouvons allez plus loin, car nos statistiques n'indiquent pas la date de naissance), mais ne sort pas directement de l'école pour $87 \%$ des cas (figure 4). Le mythe de l'étudiant surdoué " montant sa boîte " au sortir de l'école ne correspondant pas complètement au cas de la logistique.

Il existe aussi un volant de 20 à $27 \%$ de startupers qui en sont à leur troisième startup. Ainsi environ un quart des startupers logistiques sont-ils en réalité des entrepreneurs professionnels qui passent d'un projet à un autre en vue d'atteindre le succès. Cela s'intègre à l'idée californienne que l'échec n'est pas rédhibitoire, mais au contraire formateur, et qu'un entrepreneur ne peut que continuer à aller de l'avant (Krauss, 2009). Pour la plupart d'entre eux, la logistique n'est qu'un nouveau champ d'action pour leurs talents entrepreneuriaux (Berkane, 2007). La troisième catégorie, celle des anciens salariés est la plus fortement représentée. Elle atteint $60 \%$ dans la dernière activité. On voit ainsi que le passage par le salariat reste encore le parcours le plus classique pour fonder son entreprise. Cependant, les personnes ayant un emploi salarié dans leur avant-dernière activité ne sont que $47 \%$ (figure 4).

$\mathrm{Au}$ terme de ce premier balayage, on peut ainsi identifier trois profils chez les startupers : le jeune diplômé, le « serial entrepreneur » et le salarié. 

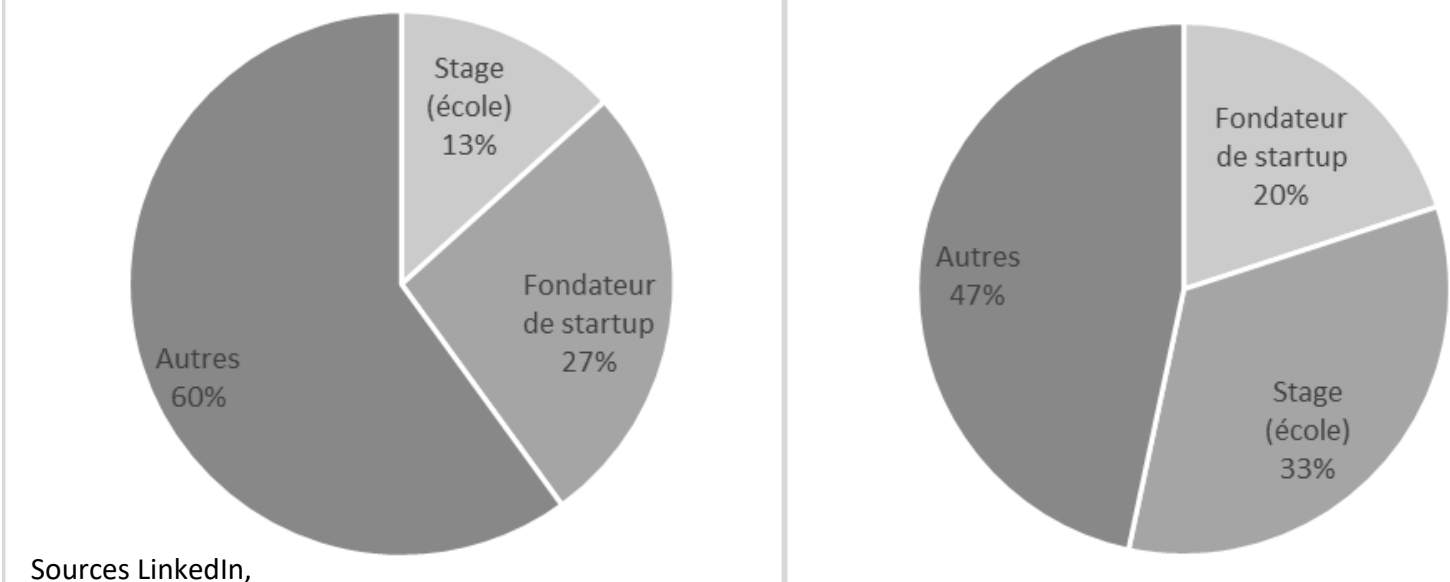

traitements personnels

Figure 4. Avant-dernier emploi (gauche) et dernier emploi (droite) des startupers

Concernant les secteurs d'activités, la logistique n'apparait qu'à hauteur de $10 \%$ dans le dernier emploi. C'est, par exemple, 3\% de moins que la finance, secteur pourtant très éloigné de la logistique. Ceux-ci viennent y travailler et innover, mais n'en sont pas issus. Au sujet de l'avant-dernier emploi, ce constat est encore plus frappant, avec une absence totale de lien avec la logistique (figure 5).

Le deuxième constat qui s'impose est la prégnance des métiers du web chez ces startupers de la logistique. Avec 17\% dans l'avant-dernier emploi et 34\% dans le dernier emploi, c'est ce secteur qui fournit le plus gros contingent (figure 5). On peut l'analyser comme la confirmation du caractère crucial de la proximité avec les nouvelles technologies et de la capacité à les manier. Autant que la maîtrise stricto sensu des outils technologiques, c'est certainement la capacité de ces diplômés d'écoles de commerce ou d'universités sélectives à saisir le potentiel marchand de ces outils qui leur donne un avantage comparatif décisif.
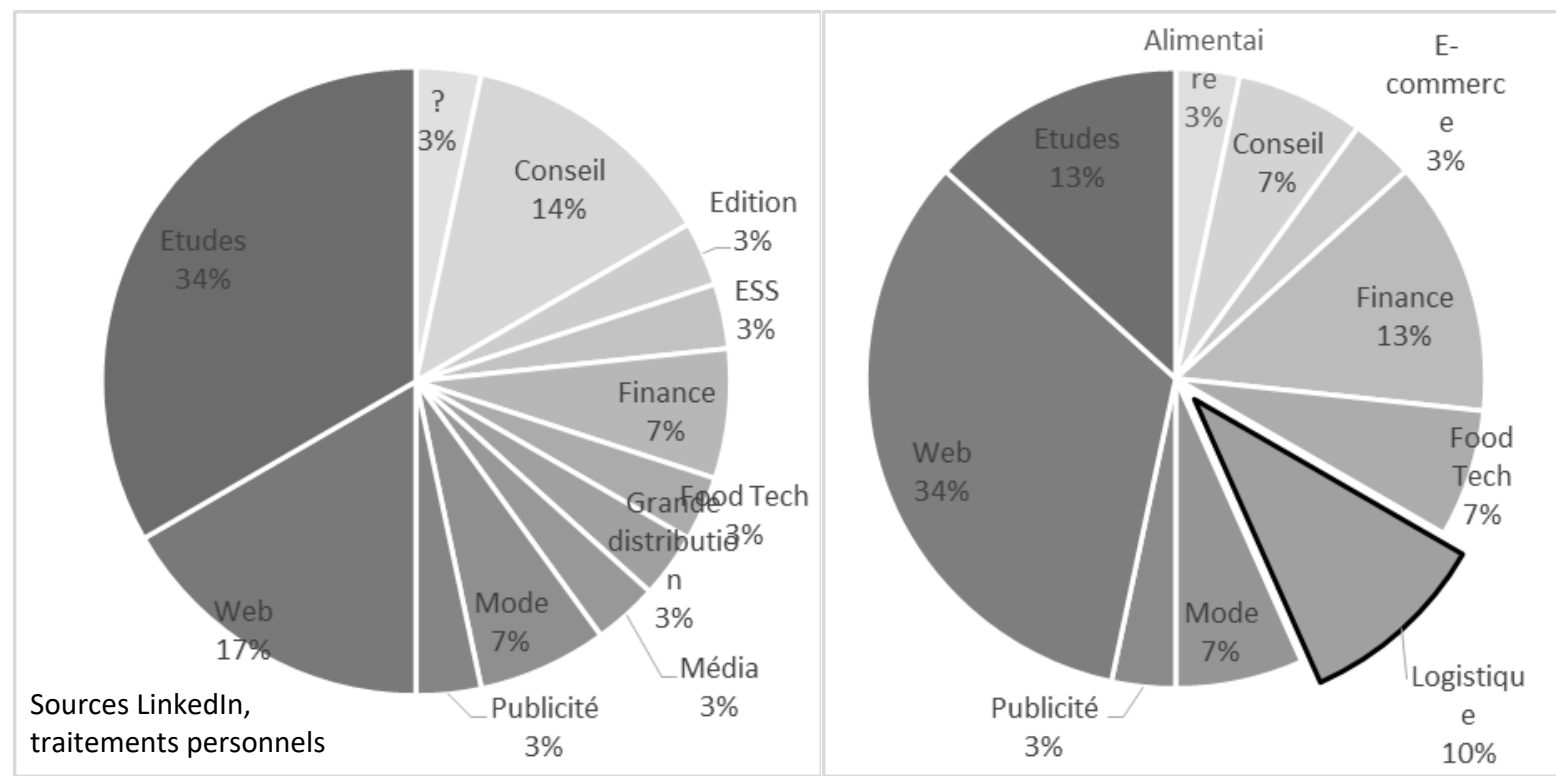

Figure 5. Domaine d'activité de l'avant-dernier emploi (gauche) et du dernier emploi (droite) des startupers

Finalement, il apparaît que derrière les enjeux d'uberisation, se joue aussi un changement potentiel de la sociologie des dirigeants logistiques. L'uberisation génère ainsi un renouvellement des groupes dominants au sein du secteur logistique. 


\section{Une disruption destructrice d'emplois?}

A travers cette analyse, un constat d'impose : les emplois de chauffeurs-livreurs ne sont pas directement menacés par l'uberisation. Leur statut juridique pourrait être modifié, mais pas le volume d'activités à traiter. Inversement, la numérisation et de l'automatisation de l'économie semble plutôt amener à une destruction d'emplois intermédiaires (supprimant des tâches autrefois accomplies par des humains), notamment ceux des responsables des tournées. L'exemple de Citodi, startup logistique proposant du dispatching ${ }^{21}$, est à ce titre limpide.

"On est les seuls à faire du dispatch automatique. Traditionnellement, il y a un dispatcheur humain qui répartissait les livreurs et les courses de la meilleure des façons possibles. Avec Citodi, on l'a automatisé et donc remplacé. »

(Entretien, cofondateur de Citodi, 03/05/2016)

Ainsi, l'exemple de Citodi montre que l'uberisation peut certes détruire des emplois, mais à des niveaux de responsabilité que l'on ne soupçonnait pas forcément. On retrouve ici les prévisions de nombreux économistes qui insistent sur le fait que cette révolution technologique ne va pas épargner les emplois intermédiaires ${ }^{22}$.

En se concentrant sur le dernier kilomètre, l'uberisation en cours de la logistique remet en cause l'organisation actuelle des transports légers et les métiers qu'ils recouvrent. Cependant, les solutions apportées par ces nouveaux outils s'inscrivent largement dans la logique d'externalisation qui structure déjà ce secteur et qui pèse fortement sur les conditions de travail des chauffeurs-livreurs. C'est pourquoi, plutôt que d'opposer ces deux systèmes, il convient de souligner les continuités entre eux, les deux se succédant chronologiquement. L'uberisation serait le déploiement final d'une promesse contenue dans le processus de libéralisation du transport routier entamée dans les années 1980 en France et en Europe : la recherche de flexibilité jusqu'à la disparition du salarié avec tous les avantages qu'il avait pu acquérir (Kyrou, 2015). En ce sens, cette remise en cause aura finalement peut-être autant d'impacts sur les dirigeants et les donneurs d'ordre de cette activité déjà largement sous-traitée que les conditions de travail des livreurs eux-mêmes.

\section{L'interface entre grande logistique et livraisons urbaines}

En présentant le paysage de la logistique francilienne, nous avons insisté sur une dichotomie structurante à la fois en termes de métiers et d'un point de vue territorial entre le domaine des livraisons dans les zones urbaines denses et celui des entrepôts du périurbain. Or, si la logistique du dernier kilomètre est potentiellement uberisable à court terme, notre travail montre que le reste des activités logistiques n'est pas concerné dans l'immédiat par une telle remise en question. En effet, ces dernières demandent trop de capitaux et de moyens techniques qui ne sont pas facilement disruptés par des startups : il est difficile d'imaginer un particulier mettre son entrepôt ou son poids lourd à disposition sur une application mobile. En ce sens, l'uberisation des livraisons urbaines renforce la dichotomie déjà existante avec la grande logistique, celle des entrepôts.

A travers ce constat, apparaît une potentielle fragmentation sociale entre une grande logistique, qui ne serait pas impactée et qui connaîtrait ainsi le maintien d'une forme de salariat (avec un recours important au travail intérimaire) et celle du dernier kilomètre, massivement uberisée. Nous aurions face à face deux mondes ouvriers, l'un salarié, l'autre uberisé, dans un même secteur d'activité. Cette fragmentation peut être porteuse à l'avenir de contestations sociales et de compétition entre les deux

\footnotetext{
${ }^{21}$ Dans une fonction logistique, il s'agit de trier, orienter et envoyer des marchandises à différents destinataires.

${ }^{22}$ The Future of Jobs, World Economic Forum, Davos, janvier 2016.

(c) 2017 ISTE OpenScience - Published by ISTE Ltd. London, UK - openscience.fr 
groupes. Cette fragmentation est également territoriale entre une logistique des grandes périphéries et une logistique des zones urbaines denses.

L'uberisation soulève toutefois la question de la frontière, territoriale et fonctionnelle, entre ces deux logistiques : jusqu'à quel point l'uberisation peut-elle remonter la chaîne logistique ? Où se situe et se situera l'interface entre les deux logistiques ? Autrement dit, où les livreurs ubérisés viendront-ils chercher les colis à livrer? Plusieurs solutions de distribution urbaine sont aujourd'hui utilisées. Actuellement, depuis les grands entrepôts de périphérie, les marchandises sont généralement envoyées soit directement vers les magasins, soit vers des sites de tri de plus petite ampleur, appelés agences de messagerie, où s'organisent les tournées. Plus précisément, ces sites de tri aiguillonnent les marchandises en direction soit des domiciles des particuliers, soit de points relais où les clients peuvent retirer eux-mêmes leurs colis. Ces différents sites, le grand entrepôt, l'agence de messagerie, le point relais ou même le magasin sont autant d'interfaces potentielles entre les deux logistiques, situées plus ou moins en amont de la chaîne logistique.

Aujourd'hui, très peu de start-ups envoient leur livreurs uberisés à la source, à l'entrepôt, chercher des colis, ni même dans les agences de messagerie, qui restent la chasse gardée des grands groupes de livraison, DHL ou Chronopost, et de leurs sous-traitants selon les logiques que nous avons présentées précédemment. La majorité des livraisons uberisées fonctionne à partir des points relais et plus encore des magasins. Il s'agit d'une stratégie délibérée. "Ni la ville ni la logistique ne vont tenir le choc face à l'inflation des volumes de marchandises qui circulent [...], Nous sommes l'anti-Amazon : pas d'entrepôt, la ville est notre stock »expliquait Serge Alleyne, fondateur de TokTokTok en octobre 2015 aux Echos ${ }^{23}$. Cette stratégie consiste à considérer la ville comme un entrepôt où l'on se sert sans financer de lourds équipements de logistique urbaine. Cependant, cette approche peut vite atteindre ses limites si l'uberisation se développe et absorbe de nombreuses facettes du transport de marchandises en ville. La pression qu'exerceront les livreurs sur les stocks en magasin sera trop importante; une nouvelle solution devra être trouvée car les stocks en magasin coûtent cher. C'est ce qui s'est passé il y a quelques années avec le développement du e-commerce alimentaire. Au départ le "picking » ${ }^{24}$ en magasin était une bonne solution pour constituer les paniers commandés, mais avec la croissance des commandes de courses en ligne, il a fallu repenser une partie de la logistique et préparer ces commandes au sein des entrepôts des distributeurs. L'analogie nous pousse à considérer qu'un maillon va manquer pour lier les deux logistiques. Il prendrait la forme d'un entrepôt urbain qui viendrait relayer les stocks en magasin qui sont insuffisants.

Or, le marché de l'immobilier logistique ne propose pas encore un tel produit (Raimbault, 2016), et les initiatives publiques visant à implanter des espaces logistiques en zone urbaine dense ont rarement été couronnées de succès (Dablanc, 2013). Face à ces difficultés, des entreprises commencent à réfléchir à des solutions que l'on pourrait qualifier de solutions de contournement. C'est notamment ce que recherche Citodi qui veut ainsi permettre à des poids lourds de charger des marchandises depuis les grandes plateformes de périphérie et de fixer un point de rendez-vous sécurisé en entrée de ville, par le biais d'un algorithme maison, avec des livreurs, potentiellement uberisés, qui emporteraient les colis correspondant à leur zone géographique et en assureraient la livraison finale ${ }^{25}$.

"C'est l'algorithme qui trouve les points d'intérêt où garer les camions. Cela pourrait-être selon nous un moyen de contourner l'absence de foncier logistique en centre-ville » (Entretien cofondateur de Citodi, 07/05/2016).

\footnotetext{
23 Paul Molga, « Demain tous livreurs ! » Les Echos, octobre 2015, [URL : http://www.lesechos.fr/idees-debats/sciencesprospective/021398769982-logistique-demain-tous-livreurs-1164739.php?mhm2eX1thiump8XM.99; consulté le 06/06/2016].

${ }^{24}$ « Préparation des commandes qui consiste à prélever les Unités de Vente [UV] dans le stock. Elles sont rassemblées pour constituer les colis » in Ropital C., Vétois P., Glossaire du e-commerce et de la logistique, IAU IdF, [consulté le 04/04/2016].

${ }^{25}$ Pour cette initiative, Citodi a été récemment retenue dans le cadre de l'appel à projet « Logistique urbaine durable » de la Ville de Paris. 
A l'image de la solution proposée par Citodi, l'absence de produits immobiliers urbains pour la logistique pourrait faire émerger des solutions innovantes d'interfaces articulant plus ou moins le virtuel (le logiciel) et la mobilité (des points de rendez-vous). Autrement dit, l'uberisation des livraisons pourrait conduire à une forme d'uberisation de l'interface entre grande logistique et logistique urbaine qui ne serait pas sans effet sur l'usage de la voirie et des espaces publics urbains.

\section{Conclusion}

Une uberisation de la logistique est à l'œuvre : les nouvelles technologies de l'information jouent aussi un rôle majeur en matière de transport de marchandises. Fonction essentielle à l'économie de la métropole parisienne, la logistique va probablement connaitre une uberisation partielle qui va principalement concerner, dans un premier temps, les livraisons les plus urbaines. En ce sens, l'uberisation de ce maillon de la chaîne logistique s'inscrit dans la lignée du processus plus ancien d'externalisation et de sous-traitance de cette activité. Ainsi, l'uberisation observée apparait-elle davantage comme une mise à jour numérique de la logistique du dernier kilomètre que comme une véritable disruption.

Cependant, il convient de rester prudent, car l'actualité récente nous prouve que les jeunes pousses du secteur n'ont pas répondu à toutes les questions qu'elles soulèvent. Ainsi Take Eat Easy, évoquée plus haut, a été mis en redressement judiciaire fin juillet 2016 (en même temps que deux autres startups uberisées mais non logistiques ${ }^{26}$ ) car la rentabilité n'était pas au rendez-vous malgré des volumes traités importants. Les investisseurs se sont inquiétés et la dernière levée de fonds s'est révélée être un échec. Dans ces conditions, l'entreprise s'est arrêtée. Cela nous appelle à la vigilance car le secteur va surement connaître une floraison de startups qui ne pourront toutes survivre. Un phénomène de concentration se fera nécessairement jour, pour qu'un acteur puisse l'emporter. La rentabilité est encore le point le plus critique de toutes ces startups. Pour beaucoup de startups, le but du jeu actuel est de réussir à lever des fonds (malgré l'absence de rentabilité) le plus longtemps possible, la rentabilité n'intervenant que lorsqu'ils seront devenus les acteurs dominants du secteur suivant la logique du « winner takes all » (pouvant alors relever leur prix).

Cette instabilité montre que ce phénomène n'en est qu'à ses prémices. Il est donc difficile d'en tirer des conclusions définitives. Une piste intéressante pour préciser les enjeux probables de l'uberisation logistique serait d'obtenir des données plus précises sur les chauffeurs-livreurs franciliens. Cela permettrait d'appréhender les possibilités de déversement depuis une activité de sous-traitants à une activité d'indépendants uberisés. Par ailleurs, les destructions d'emplois ne sont pas forcément là où on les attendait: si les chauffeurs-livreurs ne verront pas leurs activités décroître, certains emplois intermédiaires sont, eux, à terme, menacés. De plus, la sociologie des dirigeants pourrait être bouleversée. La question juridique, par ailleurs, n'est pas encore éclaircie car, cela était manifeste concernant d'Uber Pop, le législateur est appelé à prendre position sur ces sujets de société tôt ou tard.

Finalement, au-delà du seul cas de la logistique, cette étude de cas met en évidence certaines conditions favorisant l'uberisation d'un secteur économique, notamment en termes d'intensité technique et capitalistique et d'importance des logiques de sous-traitance. Elle illustre aussi l'idée que l'uberisation n'est nécessairement une rupture radicale, voire une révolution comme on aime à la présenter. Elle pourrait être tout autant une dynamique s'inscrivant dans la continuité de tendances déjà anciennes. C'est là une piste à explorer pour de futures recherches.

Enfin, deux autres perspectives de recherche s'esquissent à travers notre étude de cas. Tout d'abord, nous avons montré tout l'intérêt d'analyser non seulement les impacts de l'uberisation proprement dite, mais aussi les impacts sur les activités liées qui ne sont pas directement concernées par cette dynamique ainsi que la problématique de l'interface entre ces deux domaines. Enfin, l'uberisation, qui

\footnotetext{
${ }^{26}$ Save.co et Chics Types ont été mises en redressement judiciaire à l'été 2016. 
concerne pour l'instant des activités économiques à caractère très urbain, soulève également des enjeux cruciaux sur la production et l'utilisation des espaces urbains, ce qui pourrait susciter l'émergence de nouvelles politiques urbaines.

\section{Bibliographie}

BARBA C., (2011), 2020: la fin du e-commerce ... ou l'avènement du commerce connecté, Rapport FEVAD, FEVAD.

BERKANE A. (2007), Schumpeter et la sociologie économique : le cas de l'entrepreneur, Working Paper, Nice, Gredeg.

BOUTILLIER S., TIRAN A., (2016) « La théorie de l'entrepreneur, son évolution et sa contextualisation », Innovations, $\mathrm{n}^{\circ} 50$, pp. 211-233.

CGEDD, (2012), «Un essai de mesure de l'impact de la logistique sur le transport routier de marchandises », Etudes et documents, $n^{\circ} 66$, mai.

CHARRIN E. (2013), La course ou la ville, Paris, Seuil, coll « Raconter la vie ».

CHRISTENSEN C. (2013) (réédition), The Innovator's Dilemma, When New Technologies Cause Great Firms To Fails, Harvard Business Review Press.

DABLANC, L. (2013), « City Logistics », in Rodrigue J.-P., Notteboom T., Shaw J. (dir.), The SAGE Handbook of Transport Studies, SAGE, pp. 119-139.

DABLANC, L., FREMONT A. (dir.) (2015), La métropole logistique. Le transport de marchandises et le territoire des grandes villes, Armand Colin

DURAND B., GONZALES-FELIU, J., F. HENRIOT (2010), « La logistique urbaine, facteur clé de développement du B to $\mathrm{C} »$, Logistique \& Management, 18(2), pp. 7-19.

FEVAD (2015), Chiffres clés 2015, FEVAD.

FRIEDMAN G. (1956), Le Travail en miettes, Paris, Gallimard.

FILIPPOVA D. (dir.) (2016), Société collaborative, la fin des hiérarchies, Paris, Rue de l'Echiquier.

GABORIEAU D. (2012), « Le nez dans le micro ». Répercussions du travail sous commande vocale dans les entrepôts de la grande distribution alimentaire, La nouvelle revue du travail [En ligne], 1|2012. URL : http://nrt.revues.org/240 [consulté le 15/09/2016]

HESSE M. (2008), The City as a Terminal. The Urban Context of Logistics and Freight transport, Aldershot, Ashgate.

JOUFFE Y. (2008), « Les chauffeurs-livreurs parisiens : la place de la sous-traitance dans la chaîne logistique parisienne. Rapport de recherche », rapport final à l'INRETS.

KRAUSS G. (2009), Les jeunes entreprises pionnières face à l'incertitude : la construction sociale de l'échec, Revue Française de Socio-Économie, 1/2009 (n 3), 169-186.

KYROU A., (2015), «L’uberisation est un populisme », Multitudes nº61, pp. 106-113.

LIBESKIND J. (2015), La logistique urbaine, les nouveaux modes de consommation et de livraison, FYP, Paris

LEVRATTO N.,TESSIER L. (2016), « Business Angels et performance d'entreprise, une analyse empirique sur données françaises », dans Innovations n49 pp.141-176

MARIOTTE H. (2007), L’emploi dans la fonction logistique en France, Notes du SESP, n¹6, 1-4.

MORGANTI E. (2013), « Les achats sur internet et la livraison. Quelle logistique urbaine pour les colis ? Projet PUCA, IFSTTAR.

INSEE (2015), 375000 emplois salariés dans la logistique en Ile-de-France, Insee Analyses nº 13.

RAIMBAULT N. (2014), Gouverner le développement logistique de la métropole : périurbanisation, planification et compétition métropolitaine, le cas du bassin parisien et éclairages étrangers, sous la direction d'Antoine Frémont, thèse de doctorat, Université Paris-Est.

RAIMBAULT N. (2016), «Ancrer le capital dans les flux logistiques: la financiarisation de l'immobilier logistique », Revue d'Économie Régionale \& Urbaine 1/2016 (Février), pp. 131-154.

REME-HARNAY P. (2012) «Les logiques de sous-traitance urbaine », actes du séminaire Pour un renouveau de la logistique urbaine. 
REME-HARNAY P., CRUZ C., DABLANC L. (2014), « La sous-traitance de la messagerie urbaine : logiques économiques et rapports de dépendance », Economie et société, Série AB : Socio Economie du Travail, 2014, n³6, pp 1473-1512.

SCHUMPETER J. (1942), Capitalisme, socialisme et démocratie, Paris, Payot.

SOUFRON J.-B. (2015), « Uber ou les taxis, qui doit s'adapter? », Esprit 8/2015 (Août-septembre), pp. 204-208.

TEBOUL B. (2016), «L'Uberisation, l'automatisation... Le travail, les emplois de la seconde vague du numérique ». Big Data et Emploi : Séminaire en Economie, Compiègne

TRANCHANT L. (2015), «Nouveaux emplois, nouvelle condition? Les professions de la logistique au cœur des recompositions du groupe ouvrier en France (1982-2012) », Séminaire Transport et Mondes Professionnels, 19 novembre 2015, Université Paris-Est.

\section{Annexes}

\begin{tabular}{|c|c|c|}
\hline Entreprises & Fonction de la personne interrogée & $\begin{array}{c}\text { Date de } \\
\text { l'entretien }\end{array}$ \\
\cline { 3 - 3 } Citodi & Fondateur & $03 / 05 / 2016$ \\
\hline Take Eat Easy & Livreurs & $25 / 04 / 2016$ \\
\hline Chronotruck & Responsable commercial & $06 / 05 / 2016$ \\
\hline Deliver.ee & Fondateur & $13 / 05 / 2015$ \\
\hline Colisweb & Fondateur & $20 / 05 / 2015$ \\
\hline UPS & Responsable Relations publiques & $16 / 06 / 2015$ \\
\hline
\end{tabular}

Tableau 2. Entretiens réalisés 


\begin{tabular}{|c|c|c|c|c|c|c|}
\hline $\begin{array}{l}\text { Entreprise } \\
\text { actuelle }\end{array}$ & $\begin{array}{l}\text { Entreprise } \\
\text { précédente } \\
\quad \# 1\end{array}$ & Secteur & $\begin{array}{c}\text { Entreprise } \\
\text { précédente \#2 }\end{array}$ & Secteur & Formation & $\begin{array}{c}\text { Type de } \\
\text { formation }\end{array}$ \\
\hline Deliveroo & Deliver.ee & Logistique & Publicis Conseil & Conseil & $\begin{array}{c}\text { Ecole du } \\
\text { Multimédia }\end{array}$ & $\begin{array}{c}\text { Ecole } \\
\text { spécialisée }\end{array}$ \\
\hline Deliver.ee & $\begin{array}{l}\text { Publicis } \\
\text { Conseil } \\
\end{array}$ & Conseil & Publicis Modem & Conseil & HEC & $\begin{array}{c}\text { Ecole de } \\
\text { commerce }\end{array}$ \\
\hline Drivoo & $\begin{array}{c}\text { THE } \\
\text { STÜDNET }\end{array}$ & Web & www.jetbee.com & Web & IMERIR & $\begin{array}{c}\text { Ecole } \\
\text { spécialisée }\end{array}$ \\
\hline Botfuel & Drivoo & Logistique & BNP Paribas & Finance & HEC & $\begin{array}{c}\text { Ecole de } \\
\text { commerce }\end{array}$ \\
\hline Colisweb & $?$ & $?$ & $?$ & $?$ & $\begin{array}{c}\text { Skema } \\
\text { Business } \\
\text { School }\end{array}$ & $\begin{array}{l}\text { Ecole de } \\
\text { commerce }\end{array}$ \\
\hline Stuart & Resto In & Food Tech & City Cake & Food Tech & ESCP & $\begin{array}{c}\text { Ecole de } \\
\text { commerce }\end{array}$ \\
\hline Stuart & Resto In & Food Tech & $?$ & $?$ & $\begin{array}{c}\text { The } \\
\text { American } \\
\text { University of } \\
\text { Paris }\end{array}$ & $\begin{array}{l}\text { Université } \\
\text { sélective }\end{array}$ \\
\hline Stuart & Google & Web & Sparrow Mail & Web & HEC & $\begin{array}{l}\text { Ecole de } \\
\text { commerce }\end{array}$ \\
\hline Convargo & Vaniday & Web & $\begin{array}{c}\text { Nova Founders } \\
\text { Capital }\end{array}$ & Finance & EDHEC & $\begin{array}{c}\text { Ecole de } \\
\text { commerce }\end{array}$ \\
\hline Chronotruck & $\begin{array}{c}\text { Groupe } \\
\text { Rodolphe } \\
\text { Allard }\end{array}$ & Logistique & Monoprix & $\begin{array}{c}\text { Grande } \\
\text { distribution }\end{array}$ & $\begin{array}{c}\text { ISG } \\
\text { International }\end{array}$ & $\begin{array}{l}\text { Ecole de } \\
\text { commerce }\end{array}$ \\
\hline Chronotruck & Evaneos & Web & FIA-NET & Web & $?$ & $?$ \\
\hline Chronotruck & EdenPay & Web & BDMJ Holding & & Science Po & $\begin{array}{l}\text { Université } \\
\text { sélective }\end{array}$ \\
\hline TokTokTok & Nomao & Web & Multivea & Web & IUT Vélizy & Université \\
\hline TokTokTok & Teads & Publicité & Teads & Publicité & $\begin{array}{l}\text { Macquarie } \\
\text { University }\end{array}$ & $\begin{array}{l}\text { Université } \\
\text { sélective }\end{array}$ \\
\hline $\begin{array}{c}\text { GoGo } \\
\text { RunRun }\end{array}$ & Rives & Mode & KPMG & Conseil & $\begin{array}{c}\text { Paris } \\
\text { Dauphine }\end{array}$ & $\begin{array}{l}\text { Université } \\
\text { sélective }\end{array}$ \\
\hline Citodi & Adsvisers & Web & Ubudu & Web & EDHEC & $\begin{array}{c}\text { Ecole de } \\
\text { commerce }\end{array}$ \\
\hline Phenix & $\begin{array}{l}\text { Merryl } \\
\text { Lynch }\end{array}$ & Finance & $?$ & $?$ & ESSEC & $\begin{array}{c}\text { Ecole de } \\
\text { commerce }\end{array}$ \\
\hline Phenix & Ballistic & Mode & Eqosphere & ESS & ETNA & $\begin{array}{c}\text { Ecole } \\
\text { spécialisée }\end{array}$ \\
\hline Fretlink & IBCorp & Web & $?$ & $?$ & Paris School & Ecole de \\
\hline
\end{tabular}




\begin{tabular}{|c|c|c|c|c|c|c|}
\hline & & & & & of Economics & commerce \\
\hline Fretlink & IBCorp & Web & $?$ & $?$ & $\begin{array}{c}\text { ISG } \\
\text { International }\end{array}$ & $\begin{array}{l}\text { Ecole de } \\
\text { commerce }\end{array}$ \\
\hline Wing & $\begin{array}{c}\text { Rémy } \\
\text { Cointreau }\end{array}$ & Alimentaire & $?$ & $?$ & ESCE & $\begin{array}{c}\text { Ecole de } \\
\text { commerce }\end{array}$ \\
\hline Wing & $\begin{array}{c}\text { Vente } \\
\text { privée.com }\end{array}$ & $\begin{array}{c}\text { E- } \\
\text { commerce }\end{array}$ & 2Point1 & Mode & ESDES & $\begin{array}{l}\text { Ecole de } \\
\text { commerce }\end{array}$ \\
\hline Wing & Void Clash & Web & 2Point2 & Mode & EPITECH & $\begin{array}{c}\text { Ecole } \\
\text { spécialisée }\end{array}$ \\
\hline Délivreurs & $\begin{array}{c}\text { Argon } \\
\text { Consulting } \\
\end{array}$ & Conseil & PWC & Conseil & Supélec & $\begin{array}{c}\text { Ecole } \\
\text { d'ingénieur }\end{array}$ \\
\hline Cubyn & $\begin{array}{l}\text { Rocket } \\
\text { Internet }\end{array}$ & Finance & $?$ & $?$ & HEC & $\begin{array}{l}\text { Ecole de } \\
\text { commerce }\end{array}$ \\
\hline Shippeo & $\begin{array}{l}\text { Vermeer } \\
\text { Capital }\end{array}$ & Finance & Ateliers Volume & Edition & HEC & $\begin{array}{l}\text { Ecole de } \\
\text { commerce }\end{array}$ \\
\hline Shippeo & $\begin{array}{l}\text { Vermeer } \\
\text { Capital }\end{array}$ & Finance & TF1 & Média & Science Po & $\begin{array}{c}\text { Université } \\
\text { sélective }\end{array}$ \\
\hline Shippeo & $?$ & $?$ & $?$ & $?$ & Science Po & $\begin{array}{l}\text { Université } \\
\text { sélective }\end{array}$ \\
\hline Shippeo & $?$ & $?$ & $?$ & $?$ & Science Po & $\begin{array}{c}\text { Université } \\
\text { sélective }\end{array}$ \\
\hline Shippeo & $?$ & $?$ & $?$ & $?$ & Science Po & $\begin{array}{l}\text { Université } \\
\text { sélective }\end{array}$ \\
\hline
\end{tabular}

Tableau 3. Base de données sur les startupers (sources : Linkedln, traitements personnels) 